\title{
Adrenal Injuries and Incidentalomas in Trauma Patients at an Urban Trauma Centre
}

\author{
Stacey Woodruffa, Meei Yeung ${ }^{\mathrm{a}, \mathrm{b}}$, Simon Grodski ${ }^{\mathrm{a}}$, \\ William Johnson ${ }^{\text {a }}$, Jonathan Serpell ${ }^{\text {a }}$
}

\begin{abstract}
Background: Trauma patients are frequently evaluated by abdominal computed tomography. The prevalence of adrenal incidentalomas is $4 \%$ of the general population, increasing with age. The aim of this study was to examine the incidence and follow-up of adrenal lesions in an adult trauma centre.
\end{abstract}

Methods: A retrospective review was performed of all trauma admissions to an urban trauma centre in Melbourne, Australia between August 2000 and October 2009 with a discharge diagnosis including adrenal injury, mass, or adenoma.

Results: From August 2000 through October 2009, there were 45,576 patients admitted to the Alfred Hospital Trauma Unit, 142 $(0.31 \%)$ patients had adrenal injuries and $26(0.06 \%)$ patients had adrenal incidentalomas. Of the patients with adrenal injuries, the male-to-female ratio was $3: 1$. Blunt trauma was responsible in $99 \%$ of patients. Right-sided injuries were commoner (73\%). Left-sided lesions were present in 19\%, and bilateral injuries in $5 \%(\mathrm{P}<$ $0.0001)$. Adrenal injury was identified as haemorrhage or haematoma in $95 \%$. In the adrenal injury group, there was the $13 \%$ mortality. Follow-up imaging was not performed in $50 \%$ of patients; while another $30 \%$ only had imaging within 2 weeks, while still hospitalised. Of the 26 patients with incidentalomas, the male-to-female ratio was 1.6:1. All were blunt trauma patients, $69 \%$ of identified masses were left-sided lesions, $23 \%$ right-sided, and $7.7 \%$ were bilateral. Repeat imaging was obtained in $58 \%$ of patients, while the remainder was lost to follow-up. Biochemical investigations were performed in $54 \%$. One patient had phaeochromocytoma, and 13 patients had benign incidentalomas. Three patients underwent adrenalectomy: one at the time of trauma laparotomy (benign cortical adenoma); one 5 months post-trauma (phaeochromocytoma); and

Manuscript accepted for publication October 4, 2012

${ }^{a}$ Monash University Endocrine Surgery Unit, Alfred Hospital, Commercial Rd, Prahran 3181, Victoria, Australia

${ }^{\mathrm{b}}$ Corresponding author: Meei Yeung, Department of Surgery, Alfred

Hospital, Commercial Rd, Prahran 3181, Victoria, Australia.

Email: yeung.m@bigpond.com

doi: http://dx.doi.org/10.4021/jcs108w the final patient 3 months post-trauma (benign vascular epithelial cyst).

Conclusions: With increased use of abdominal CT in the evaluation of trauma patients, adrenal lesions are increasingly likely to be found. Follow-up of these lesions in this series has been variable. The development of a protocol for adrenal lesions in trauma patients may improve follow-up care.

Keywords: Adrenal haematoma; Adrenal injury; Adrenal incidentaloma; Adrenal trauma

\section{Introduction}

Computed tomography (CT) has become the primary diagnostic modality in the evaluation of moderate to severely injured trauma patients [1]. With the increased use of abdominal CT in the evaluation of trauma patients, incidental adrenal lesions are detected more frequently [1-4]. It is estimated that traumatic adrenal injuries are present in $0.2-2 \%$ of all trauma admissions [5, 6]. Although rare, adrenal gland injury (AGI) can be potentially harmful if undetected [1]. Haemorrhage into pre-existing adrenal lesions has been reported $[4,7]$. Adrenal gland injury is most often associated with multiple organ injuries, due to the significant amount of energy required to cause adrenal injury $[2-4,6]$. Significant haemorrhage requiring transfusion, and adrenal insufficiency can occur with these injuries $[2,3,6]$.

CT signs of AGI include: gland enlargement, peri-adrenal fat stranding, a high attenuation mass within or obscuring the gland, retroperitoneal haematoma, or contrast extravasation in active adrenal haemorrhage [5]. Adrenal gland injury is a marker of overall injury severity, and is associated with higher mortality $[3,4]$.

Adrenal incidentalomas, defined as clinically inapparent adrenal masses identified in the course of diagnosis or treatment for clinical conditions unrelated to the adrenal gland, are found in $4 \%$ of the general population, with the prevalence increasing with age [8]. These lesions were first described nearly 30 years ago, and understanding of their 
Table 1. Demographic Data of Adrenal Injury Group ( $N=142)$

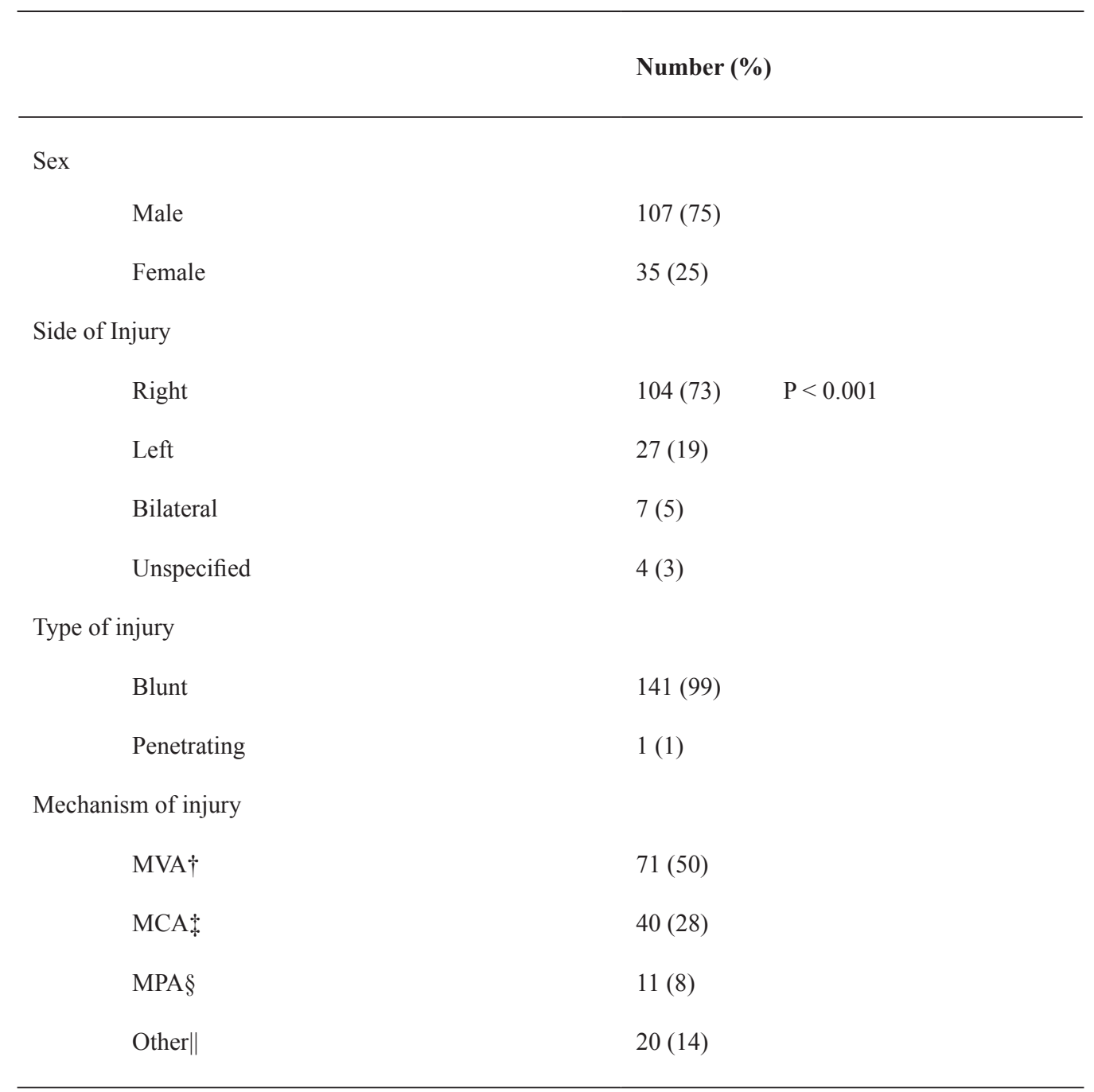

†MVA: Motor vehicle accident; ‡MCA: Motorcycle accident; §MPA: motor pedestrian accident; ||Includes falls, pedal-cycle accidents, horse-related accidents, machinery-related accidents, electricity or fire related accidents, and other transport related accidents.

impact on public health has increased significantly since that time [9-13]. The majority of these lesions are benign, nonfunctioning adrenocortical adenomas $[8,11,14]$. The prevalence of adrenocortical carcinoma (ACC) in an incidentaloma is related to mass size. ACC represents $2 \%$ of lesions less than or equal to $4 \mathrm{~cm}$ in diameter; $6 \%$ of those ranging from $4.1-6 \mathrm{~cm}$; and $25 \%$ of those greater than $6 \mathrm{~cm}[8,11,14$, 15]. The diagnosis of ACC portends poor clinical outcomes, with most studies reporting less than 50\% 5-year overall survival [8]. Malignant transformation and the development of excessive hormone production in a benign nonfunctioning adenoma are extremely rare.

Although algorithms exist regarding evaluation and follow-up of adrenal incidentalomas, there are few studies reporting appropriate evaluation and follow-up of AGI and adrenal incidentalomas in the trauma population. Thus, the aim of this study was to evaluate the incidence and follow-up of both AGI and adrenal incidentalomas in an adult trauma population and to attempt to define recommendations for management of these lesions.

\section{Materials and Methods}

A retrospective review of prospectively collected data was performed of all trauma admissions to an urban trauma centre (Trauma Database, The Alfred Hospital, Melbourne, Australia) between August 2000 and October 2009, evaluated by abdominal computed tomography (CT), with a discharge diagnosis including adrenal injury, adrenal mass, or adrenal 
Table 2. Cause of Death in Adrenal Injury Group $(\mathrm{N}=19)$

\begin{tabular}{ll}
\hline & Number (\%) \\
\hline Multiorgan failure & $7(37 \%)$ \\
Severe traumatic brain injury & $5(26 \%)$ \\
Multiple system severe injuries & $3(16 \%)$ \\
History missing or destroyed & $4(21 \%)$ \\
Total & $19(100 \%)$ \\
\hline
\end{tabular}

adenoma. These patients were extracted from health information management based on coding of diagnoses obtained from discharge summaries and the trauma database. Patients evaluated and discharged in the emergency department were not included. Data generated included demographic data, imaging characteristics, biochemical data, histology, and follow-up. Data were analyzed using chi-squared with significance set at $\mathrm{P}=0.05$.

As part of the workup of multiply injured trauma patients, CT scan evaluation of the chest, abdomen, pelvis, and thoracic and lumbar spine is undertaken. The indications for this are based on clinical findings and/or the mechanism of injury including pedestrian vs motorvehicles, motorbike accidents, motorists ejected from a vehicle, vehicle rollover and explosions.

The Alfred Hospital utilises a helical scanner with 0.4 sec rotation time, scanning from above the lung apices to below the symphysis pubis, with arterial phase from the top of the lung apices to the bottom of the lungs, and venous phase from the top of the diaphragm to the symphysis pubis. From August 2000 to June 2006 a General Electric Hi Speed ZXi scanner was used. From June 2006 to October 2009 a General Electric Lightspeed VCT scanner was used. Both scanners originate from the United States of America.

The patient is given $80 \mathrm{~mL}$ of UV370 contrast at $3 \mathrm{~mL} /$ sec. Soft tissue is scanned at $0.6 \mathrm{~mm}$ intervals, lung at $5 \mathrm{~mm}$ intervals, and bone at $0.4 \mathrm{~mm}$ intervals. Axial and coronal reformats for chest, abdomen, and pelvis are done at $7.5 \mathrm{~mm}$

Table 3. Demographic Data of Adrenal Incidentaloma Group (N = 26)

\begin{tabular}{|c|c|}
\hline & Number (\%) \\
\hline \multicolumn{2}{|l|}{ Sex } \\
\hline Male & $16(62)$ \\
\hline Female & $10(38)$ \\
\hline \multicolumn{2}{|l|}{ Side of lesion } \\
\hline Left & $18(69)$ \\
\hline Right & $6(23)$ \\
\hline Bilateral & $2(8)$ \\
\hline Mean size (cm) (Range) & $2.38(1.2-4.3)$ \\
\hline
\end{tabular}


Table 4. Demographic Data of Adrenalectomy Patients

\begin{tabular}{llll}
\hline Patient & Age & Sex & Mechanism Of Injury \\
\hline 1 & 60 & F & Motor vehicle accident - passenger \\
2 & 60 & M & Fall from a low height \\
3 & 32 & M & Motor vehicle accident - driver \\
\hline
\end{tabular}

thickness and $7.5 \mathrm{~mm}$ spacing. Axial and sagittal spine reformats are done at $2 \mathrm{~mm}$ thickness and $2 \mathrm{~mm}$ spacing.

\section{Results}

From August 2000 to October 2009, a total of 45,579 patients were admitted to the Alfred Hospital Trauma Unit. Of these patients, 6,320 (13.8\%) underwent abdominal CT for evaluation of traumatic injury. These CT scans are reviewed each morning by Consultant General Surgeons and Radiologists at the Trauma Unit handover meeting. This group of 6,320 patients makes up the study population.

AGI was found in 142 patients $(2.2 \%)$, while adrenal masses were found in $26(0.4 \%)$. The mean age was 41.4 years (range 15 - 85years) in the injury group, and 57.2 years (range 18 - 92 years) in the incidentaloma group. The maleto-female ratio was $3: 1$ in the injury group, and 1.6:1 in the incidentaloma group.

Demographic data for the adrenal injury group are presented in Table 1. All but one patient suffered blunt trauma, with motor vehicle accidents, motorcycle accidents, and motor-pedestrian accidents most frequent. AGI was identified as haemorrhage or haematoma in 95\%. Right-sided injuries were more common, present in 104 (73\%) of the 142 patients $(\mathrm{P}<0.001)$. The mean injury severity score (ISS) was 31 (range 1 - 66). The mean hospital length of stay was 16.5 days (range 1 - 132 days). The mean intensive care unit (ICU) length of stay was 11.2 days (range 0 - 95 days), with mean ventilator time of 8.5 days (range 0 - 95 days). The mortality in the AGI group was $13 \%$, with the most common cause of death multiorgan failure (Table 2).

Half of the AGI patients (71) had no follow-up imaging. Of the remaining 71 patients who did have follow-up imaging, 43 patients $(61 \%)$ had repeat imaging within 2 weeks, while still hospitalized. Of these, 67 patients had abdominal CT and 4 ultrasounds. The mean time for follow-up imaging was 1.2 weeks (range 0 - 4 weeks). Thirty-five (49\%) of these patients were shown to have resolving or resolved AGI, with the remainder showing a persistent adrenal gland abnormality. Four AGI patients underwent subsequent biochemical investigations with normal results.
Demographic data for the adrenal incidentaloma group are presented in Table 3. All patients suffered blunt trauma. Mean ISS was 15 (range 1 - 45). The mean hospital length of stay was 16 days (range 1 - 128 days). The mean intensive care unit (ICU) length of stay was 11.5 days (range 1 - 31 days), with mean ventilator time of 8.5 days (range 1 - 31 days).

Left sided lesions (69\%) were commoner than right sided lesions (23\%). The mean size was $2.38 \mathrm{~cm}$ (range 1.2 $-4.3 \mathrm{~cm})$. Of the 26 patients, 15 patients $(58 \%)$ had repeat imaging studies, 14 (54\%) had biochemical investigations, $11(42 \%)$ patients had both repeat imaging and biochemical investigations. Of the 26 adrenal incidentalomas, 14 (54\%) were followed up but 11 patients (42\%) were lost to followup.

Three patients (12\%) underwent adrenalectomy Table 4. The first patient underwent adrenalectomy at the time of trauma laparotomy and was subsequently found to have a benign $2.5 \mathrm{~cm}$ cortical adenoma. The second patient was a 60 year old male who fell from a low height, and was noted to be severely hypertensive in the emergency department, with a $4 \mathrm{~cm}$ left adrenal mass on CT. Appropriate diagnostic tests were performed based on the clinical suspicion of phaeochromocytoma. After adequate preoperative alpha blockade and blood pressure control, he underwent adrenalectomy 5 months post-trauma. The third patient underwent laparoscopic left adrenalectomy 3 months post-trauma due to a lesion size of $4.2 \mathrm{~cm}$, and was found to have a benign vascular endothelial cyst.

\section{Discussion}

Evaluation of trauma patients with abdominal CT has led to increased detection of AGI as well as adrenal incidentalomas. There are significant clinical implications associated with these lesions. Firstly, AGI must be distinguished from adrenal incidentalomas. Secondly, adrenal incidentalomas must then be evaluated for hormone overproduction and malignant potential. Thirdly, there are few studies reporting follow-up guidelines of these lesions in the adult trauma population. 


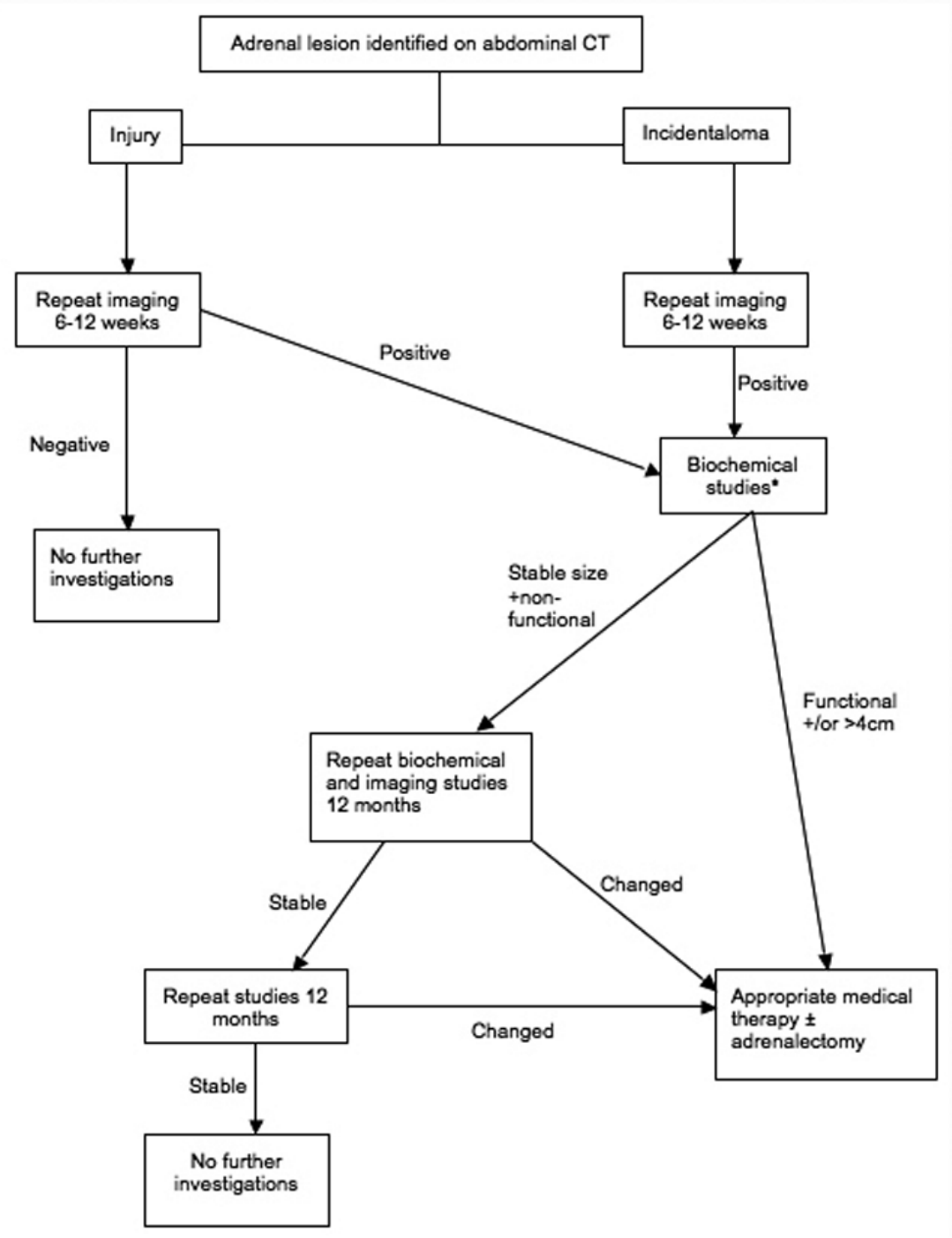

Figure 1. Algorithm for the evaluation and follow-up of incidental adrenal lesions identified during trauma workup. *Biochemical Studies include 24-hour urine catecholamines and cortisol, serum cortisol, plasma free metanephrines, aldosterone, rennin, estrogen, testosterone, dehydroepiandrosterone sulfate (DHEA-S), and potassium.

Our data show rates of AGI and adrenal incidentaloma consistent with those rates published in the literature $[2,4$, $5,8,11,15,16]$. The higher male-to-female ratio in the AGI group most likely reflects the overall male predominance in the trauma population, and is similar to published reports [24]. The mean ISS of 31 reflects that AGI is a marker of more severe trauma, as an ISS greater than 15 indicates severe trauma. This also explains the relatively high mortality of $13 \%$ in this group.

There were significantly more right-sided injuries than left. One possible explanation for this is that motor vehicles in Australia are right-hand drive. However, the rate is similar in countries with both left and right hand drive vehicles [2-4]. Theories for the preponderance of right-sided injuries are that the right adrenal gland is more easily compressed against the spine by the force of the trauma or the surrounding organs; or compression of the inferior vena cava (IVC) causes higher medullary sinusoidal pressure on the right due to the short adrenal vein that communicates directly with the IVC, thereby leading to venous congestion and tissue necrosis $[2,4,5,16]$.

Follow-up for AGI was variable in our study population, with only 71 patients $(50 \%)$ having repeat imaging at any stage post-trauma, 43 patients $(61 \%)$ underwent imaging within the first 2 weeks. Of the 36 patients who underwent repeat imaging, a persistent abnormality of the affected adrenal gland was demonstrated. Within several weeks, most AGI show radiologic evidence of healing $[5,16,17]$. Rana et 
al found that $83 \%$ of adrenal hematomas showed decreased size and attenuation when reimaged at a range of $2.5-10$ weeks [16]. If an imaging abnormality persists, one should suspect underlying pathology. Thus the remaining 36 patients having repeat imaging should have had further evaluation for adrenal incidentalomas.

Within the incidentaloma group, $42 \%$ of patients underwent both biochemical investigations and repeat imaging, most frequently CT. However, $42 \%$ of patients were lost to follow-up. Three patients underwent adrenalectomy. The first was performed at the time of trauma laparotomy. On review of the operation report, the indication for adrenalectomy was unclear, as the lesion measured $2.5 \mathrm{~cm}$ in size, and was shown to be a benign adenoma on final histopathology. This scenario is not standard practice at our institution. The second patient was found to have a phaeochromocytoma. It should be recognised that catecholamines can be elevated in the setting of trauma, and it is imperative to repeat these investigations after recovery from the traumatic injury in order to confirm the diagnosis of phaeochromocytoma.

Although a number of algorithms currently exist to aid in the management of the incidentally detected adrenal lesion $[14,18,19]$ these are not specific to the trauma population. The authors would like to propose a novel algorithm (Fig. 1) for the evaluation of adrenal lesions found on CT in the adult trauma population based on the findings of this study. An injury should have repeat imaging in 6 - 12 weeks. If the injury has resolved (negative result), then no further investigations need be performed. If there is a persistent abnormality (positive result), underlying pathology should be suspected, and the lesion should be treated as an incidentaloma. Adrenal incidentalomas should have repeat imaging studies and biochemical studies (24-hour urinary catecholamines and urinary free cortisol, serum cortisol, plasma free metanephrines, aldosterone, renin, oestrogen, testosterone, dehydroepiandrosterone sulfate (DHEA-S), and potassium) in 6 - 12 weeks. If the biochemical studies are positive, meaning the lesion is hormonally active, or if the lesion is greater than $4 \mathrm{~cm}$ in size, then the patient should be considered for adrenalectomy. If the biochemical studies are negative, and the lesion is less than $4 \mathrm{~cm}$ and unchanged in size, repeat studies should be performed at 12 months, and then again at 24 months from original diagnosis if no change has occurred. If the lesion is stable at that point, no further investigations need be performed unless symptoms develop. During the follow-up period, should the lesion increase in size or become hormonally active, the patient should be considered for adrenalectomy.

Absolute indications for surgical intervention are symptomatic, hormonally active lesions, phaeochromocytomas, malignant tumors, or lesions greater than $6 \mathrm{~cm}$ in size $[8,11]$. Smaller lesions with suspicious imaging characteristics, such as calcifications, heterogeneity, high attenuation or delayed washout on contrast CT, also warrant adrenalectomy. When indicated, the surgical approach can be either laparoscopic or open, depending on surgeon experience and patient preference. There are no prospective, randomised studies comparing the two approaches. Relative contraindications for the laparoscopic approach are ACC and large tumors with evidence of invasion which may result in a lengthy laparoscopic procedure $[8,11]$.

This study is limited by the small number of identified adrenal incidentalomas. There are several reasons for this. Firstly, the trauma population tends to be younger, and therefore the overall prevalence of adrenal incidentalomas in this group is lower 14. Second, several patients in the AGI group should have been further investigated for incidentalomas based on persistent abnormalities found on repeat imaging studies. This study is also dependent on adequate documentation of incidental findings in order to capture patients using discharge diagnosis codes. This study also excluded patients not admitted to hospital. Although this is a retrospective study, the data are prospectively collected, and all admitted patients were available for inclusion in the study.

\section{Conclusion}

With the increased use of abdominal CT in the evaluation of trauma patients, adrenal lesions are increasingly likely to be found. Follow-up of these lesions is inconsistent in the trauma population. The development and implementation of a protocol, with the involvement of the appropriate Endocrine or Endocrine Surgical units, may improve follow-up care. This will need to be substantiated in the future with data collection following implementation of this protocol.

\section{References}

1. Ekeh AP, Walusimbi M, Brigham E, Woods RJ, McCarthy $\mathrm{MC}$. The prevalence of incidental findings on abdominal computed tomography scans of trauma patients. J Emerg Med. 2010;38(4):484-489.

2. Mehrazin R, Derweesh IH, Kincade MC, Thomas AC, Gold R, Wake RW. Adrenal trauma: Elvis Presley Memorial Trauma Center experience. Urology. 2007;70(5):851-855.

3. Stawicki SP, Hoey BA, Grossman MD, Anderson HL, 3rd, Reed JF, 3rd. Adrenal gland trauma is associated with high injury severity and mortality. Curr Surg. 2003;60(4):431-436.

4. Stawicki SP, Seamon MJ, Carvalho CM, Bhoot N, Sharma R, Schrag SP, Steinberg SM. Adrenal gland injury secondary to blunt traumatic mechanisms: a marker of overall injury severity. Endokrynol Pol. 2009;60(1):2-8.

5. Sinelnikov AO, Abujudeh HH, Chan D, Novelline RA. CT manifestations of adrenal trauma: experience with 73 cases. Emerg Radiol. 2007;13(6):313-318. 
6. Gomez RG, McAninch JW, Carroll PR. Adrenal gland trauma: diagnosis and management. J Trauma. 1993;35(6):870-874.

7. Burks DW, Mirvis SE, Shanmuganathan K. Acute adrenal injury after blunt abdominal trauma: CT findings. AJR Am J Roentgenol. 1992;158(3):503-507.

8. NIH state-of-the-science statement on management of the clinically inapparent adrenal mass ("incidentaloma"). NIH Consens State Sci Statements. 2002;19(2):125.

9. Aron DC. The adrenal incidentaloma: disease of modern technology and public health problem. Rev Endocr Metab Disord. 2001;2(3):335-342.

10. Geelhoed GW, Druy EM. Management of the adrenal “incidentaloma”. Surgery. 1982;92(5):866-874.

11. Mansmann G, Lau J, Balk E, Rothberg M, Miyachi Y, Bornstein SR. The clinically inapparent adrenal mass: update in diagnosis and management. Endocr Rev. 2004;25(2):309-340.

12. Prinz RA, Brooks MH, Churchill R, Graner JL, Lawrence AM, Paloyan E, Sparagana $M$. Incidental asymptomatic adrenal masses detected by computed tomographic scanning. Is operation required? JAMA.
1982;248(6):701-704.

13. Thompson GB, Young WF, Jr. Adrenal incidentaloma. Curr Opin Oncol. 2003;15(1):84-90.

14. Young WF, Jr. Clinical practice. The incidentally discovered adrenal mass. N Engl J Med. 2007;356(6):601-610.

15. Barzon L, Sonino N, Fallo F, Palu G, Boscaro M. Prevalence and natural history of adrenal incidentalomas. Eur J Endocrinol. 2003;149(4):273-285.

16. Rana AI, Kenney PJ, Lockhart ME, McGwin G, Jr., Morgan DE, Windham ST, 3rd, Smith JK. Adrenal gland hematomas in trauma patients. Radiology. 2004;230(3):669-675.

17. van Westreenen HL, Patijn GA, Sijbrandij ES, Oosterhuis JW. [An adrenal incidentaloma in a trauma patient; a plea for expectant management]. Ned Tijdschr Geneeskd. 2007;151(49):2723-2725.

18. Boland GW, Blake MA, Hahn PF, Mayo-Smith WW. Incidental adrenal lesions: principles, techniques, and algorithms for imaging characterization. Radiology. 2008;249(3):756-775.

19. Nieman LK. Approach to the patient with an adrenal incidentaloma. J Clin Endocrinol Metab. 2010;95(9):41064113. 